\title{
FACTORES QUE INFLUYEN EN LA PARTICIPACIÓN EN CURSOS DE EDUCACIÓN CONTINUA DEL PERSONAL DE ENFERMERÍA
}

\begin{abstract}
ELEMENTS THAT INFLUENCE THE PARTICIPATION OF NURSING STAFF IN CONTINUOUS EDUCATION COURSES
\end{abstract}

Recibido: 22 junio 2017 Aceptado: 29 noviembre 2017

Correspondencia: Mtra. Olga Taboada-Aranza Dom. Lino Merino No. 411, Col. Juan Escutia 09100, Del. Iztapalapa, Ciudad de México. Correo electrónico: taao3@yahoo.com.mx

Cel. 5551864036 Tel. 2633807

Palabras clave: educación continua, capacitación, enfermería.

Key words: continuous education, training, nursing.

\section{Autores:}

Gabriela Hernández Velazco Licenciada en Enfermería. Enfermera general ISSSTE. Alumna del Curso Postécnico Gerencia y Docencia en Enfermería de la ENEI del ISSSTE.

Beatriz Ramos Arista

Licenciada en Enfermería. Enfermera general ISSSTE. Alumna del Curso Postécnico Gerencia y Docencia en Enfermería de la ENEI del ISSSTE.

Olga Taboada-Aranza

Maestra en Enseñanza Superior. Profesora Tiempo Completo de la Carrera de Cirujano Dentista de la Facultad de Estudios Superiores Zaragoza UNAM.

Julio César Cadena-Estrada

Maestro en Enfermería. Jefe de Enseñanza e Investigación del Instituto Nacional de Cardiología "Ignacio Chávez". 


\section{RESUMEN}

Introducción: la educación continua se concibe como una modalidad educativa que permite, a una persona -profesional o no-, actualizarse en un área específica para desarrollarse profesionalmente.

Objetivo: determinar los factores que influyen en la participación, en cursos de educación continua, del personal de enfermería.

Material y métodos: estudio observacional, trasversal, prolectivo y descriptivo, a una muestra por conveniencia de 136 sujetos, conformada por personal de base del área de enfermería del Hospital General Fernando Quiroz Gutiérrez del ISSSTE. Los datos fueron recolectados con un instrumento diseñado ex-profeso, auto-administrado, con una confiabilidad Alpha de Cronbach de 0.93 (0.82-0.93)

Resultados: de la participación en cursos de educación continua en los que participa el personal de enfermería, llama la atención el reporte de no asistencia a los mismos del 34\% (46) de los enfermeros(as). La antigüedad que tiene el personal de enfermería dentro del instituto, se correlaciona de manera negativa con el número de cursos tomados $(r s=-0.316, p<0.0001$ ) esto es, a mayor antigüedad, menor número de cursos tomados; mientras que a mayor nivel académico, se observa una correlacion positiva, es decir que, asisten a un mayor número de cursos ( $r$ = 0.265, $p<0.002$ ).

Conclusión: Los cursos de educación continua deben tener características de visibilidad que los hagan atractivos y, en su programación, deben considerarse las habilidades que se desea sean adquiridas.

Palabras clave: Educación continua, capacitación, enfermería.

\section{ABSTRACT}

Introduction: continuous education is conceived as an educational modality that allows a person professional or not- to update in a specific area to develop professionally.

Objective: to determine the elements that influences the participation of nursing staff in continuous education courses.

Materials and methods: an observational, transverse, prolective and descriptive study, in a convenience sample of 136 subjects comprised of nursing staff working at the General Hospital Fernando Quiroz Gutierrez of ISSSTE. Data were collected with a self-administered ex-professed instrument with a Cronbach Alpha reliability of $0.93(0.82-0.93)$.

Results: from information about the continuous education courses in which the nursing staff participates, we must highlight the non-attendance report of 34\% (46) of the nurses. The seniority of the nursing staff within the institute matches negatively with the number of courses taken ( $r s=-0.316, p<0.0001$ ), that is, the higher the number of years working, the less courses are taken; while at a higher academic level it correlates positively, that is, they attend a higher number of courses ( $r s=0.265, p<0.002$ ).

Conclusion: continuous education courses must become attractive; it is necessary to displaythe skills to be acquired in their programming.

Key words: continuous education, training, nursing.

\section{7} MMN Mhy $_{W}$ Enf Neurol Vol. 16. No. 3 septiembre - diciembre 2017 


\section{INTRODUCCIÓN}

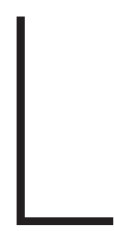

a educación es un componente de la vida, ocurre de manera continua y dinámica en el tiempo y en el espacio. El tiempo escolar no es el único espacio de formación, de aprendizaje y de cultura, el fenómeno educativo ocurre en otros lugares -las empresas, la calle, el trabajo, en los tiempos sociales y durante el ocio, entre otros - y como Boff decía "no sólo se aprende con el cerebro, ni sólo en la escuela, se aprende en la vida entera y en todas las formas de vivir".

William, mencionado por Chong, puntualiza que el conocimiento adquirido a través de la formación profesional básica tiene una vida media de 2.5 años, al final de este período, si el conocimiento no se ha actualizado a través de la educación continua se torna obsoleto, lo que genera un mal desempeño del ejercicio profesional. ${ }^{2}$

La educación continua es un proceso de formación y actualización de conocimientos y habilidades al que las personas deben someterse con el propósito de mejorar su desempeño profesional; dentro de estos se encuentra el personal de enfermería, que requiere actualizar día a día sus conocimientos en el área de la salud -ante el acelerado desarrollo de la ciencia y la tecnología- con el propósito de mejorar sus destrezas, lo que posibilita una mejor práctica profesional, en este sentido la CONAMED señala que los eventos adversos ante el cuidado de enfermería, por lo general, se presentan por la falta de conocimiento. ${ }^{3}$

Cabe señalar que, a medida que los conocimientos cambian -y con ellos la tecnología, las herramientas y los procedimientos-, la educación continua es una estrategia clave para aumentar las competencias del profesional de enfermería, y con ello, mantener la calidad en la atención. ${ }^{4}$

Hay estudios que muestran una relación directa entre la participación del personal de enfermería en cursos de educación continua, con el mejoramiento de la práctica clínica, la alta productividad, el clima organizacional y la satisfacción laboral. ${ }^{5}$
La importancia de la educación continua es evidente en una sociedad como la nuestra que, transforma su productividad en función del conocimiento acelerado y del progreso técnico, y en la cual la posibilidad y, sobre todo, la necesidad de aprender no puede darse por terminada, en este contexto, el propósito de esta investigación fue determinar los factores que influyen en la participación en cursos de educación continua del personal de enfermería.

\section{MATERIAL Y MÉTODOS}

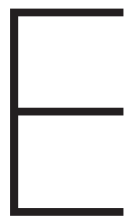

studio observacional, prolectivo, transversal, descriptivo, en el que se seleccionaron, a través de un muestreo por conveniencia, 136 integrantes del personal de enfermería del Hospital General Dr. Fernando Quiroz Gutiérrez del ISSSTE., seleccionados a través de un muestreo por conveniencia, de ellos El 16\% (22) del sexo masculino y $84 \%$ (114) del femenino; con una media de edad de 40.8 ( \pm 8.7), mínima 22, máxima 63.

Para evaluar los factores que influyen en la participación del personal de enfermería en los programas de educación continua, se aplicó el cuestionario modificado de Flores y colaboradores:? Evaluación de la participación de los cursos de educación continua del personal de enfermería, que este es un cuestionario diseñado para evaluar las siguienates dimensiones: A. Datos personales, B. Condiciones laborales, C. Participación en cursos de educación continua, con preguntas abiertas; D. Percepción acerca de los cursos de educación continua y E. Condicionantes administrativas,. Los apartados D y E tienen respuestas de opción múltiple en escala Likert con un valor de 1 a 5 puntos, donde 5 es la puntuación más alta. La confiabilidad del cuestionario obtuvo una consistencia interna un alfa de Cronbach de 0.93 (0.82-0.93).

Los datos fueron procesados en el paquete estadístico SPSS v. 20, con el cual se obtuvo la estadística descriptiva de las variables; la prueba de significancia estadística utilizada para las variables cuantitativas fue la t de Student, para las cualitativas nominales la $x^{2}$ y para las cualitativas ordinales la $r_{s}$ de Spearman con un nivel de confianza al 95\% ( $p<0.05)$. 


\section{RESULTADOS}

a razón hombre: mujer del personal de enfermería encuestado es 1:5, lo que muestra que la profesión sigue siendo más atractiva para las mujeres; de esta población el 54\% (74) son casados y 32\% (43) solteros. El número de dependientes económicos para el 37.5\% (51) es de 2, ocupando el segundo lugar con el 25.7\% (35) sólo tienen un dependiente, seis enfermeros/as reportan tener entre cuatro y cinco dependientes económicos.

El ingreso económico promedio, auto reportado, es de \$ 9,118.38 M.N. ( \pm \$ 951.19), mínimo \$8 000, máximo \$ 11 000; el promedio de años de servicio en el ISSSTE es de 12.5 ( \pm 9.4), mínimo 1, máximo 37.

El nivel académico predominante en el personal de enfermería encuestado es: técnico general con el $36.8 \%$ (50): seguido de licenciado en enfermería, con el 35.3\% (48); técnico general con especialización, 13.2\% (18); auxiliar (curso) 9.6\% (13) y, con posgrado, el $5.1 \%(6)$

Respecto al rubro, participación en cursos de educación continua, que explora el número de cursos de educación continua, en los que participa el personal de enfermería, y que buscan orientada a mantener y/o mejorar la competencia profesional, llama la atención el reporte de no asistencia a cursos, tanto internos como externos a la institución, del 34\% (46) de los enfermeros (as). En cuanto a la relación salario vs asistencia a cursos, se observa que ni el $36 \%$ (22) del personal de enfermería con ingreso $\leq \$ 8500.00$ (M.N.) o el 32\% (24) con un salario mayor no asisten a cursos, por lo que se concluye que no hubo diferencias estadísticamente significativas entre ambos grupos.

La distribución de la asistencia a cursos, respecto a ciertas características de nivel académico y condiciones laborales, se observan en el tabla 1.
Tabla 1. Número de cursos en los que participa el personal de enfermería.

Cursos tomados al año*

\begin{tabular}{|c|c|c|c|c|c|}
\hline VARIABLE & $n$ & 0 & 1062 & 30 4 & $\geq 5$ \\
\hline Edad (años) & & & & & \\
\hline$\leq 40$ & 72 & $29(21)$ & $42(30)$ & $18(13)$ & $11(8)$ \\
\hline$>40$ & 64 & ' $39(25)$ & $30(19)$ & $22(14)$ & $9(6)$ \\
\hline \multicolumn{6}{|l|}{ Sexo } \\
\hline Masculino & 22 & $14(3)$ & $59(13)$ & $27(6)$ & $0(0)$ \\
\hline Femenino & 114 & 38 (43) & $32(36)$ & $18(21)$ & $12(14)$ \\
\hline \multicolumn{6}{|l|}{ Estado civil } \\
\hline Soltero(a) & 51 & $29(15)$ & $29(15)$ & $20(10)$ & $22(11)$ \\
\hline Casado(a) & 85 & ' $37(31)$ & $40(34)$ & $20(17)$ & $3(3)$ \\
\hline \multicolumn{6}{|l|}{ Turno- } \\
\hline Matutino & 35 & $43(15)$ & $37(13)$ & $9(3)$ & $11(4)$ \\
\hline Vespertino & 47 & $28(13)$ & $38(18)$ & $25(12)$ & $9(4)$ \\
\hline Nocturno & 54 & , $33(18)$ & $33(18)$ & $22(12)$ & $12(6)$ \\
\hline \multicolumn{6}{|l|}{ Ántigüedad (años) } \\
\hline$\leq 11$ & 71 & 24 (17) & $35(25)$ & $24(17)$ & $17(12)$ \\
\hline$>11$ & 65 & $45(29)$ & $37(24)$ & $15(10)$ & $3(2)$ \\
\hline \multicolumn{6}{|l|}{ Nivel académico } \\
\hline Técnico & 81 & $45(36)$ & $32(26)$ & $16(13)$ & $7(6)$ \\
\hline Licenciatura & 48 & $19(9)$ & $42(20)$ & $25(12)$ & $14(7)$ \\
\hline Posgrado & 7 & $14(1)$ & $43(3)$ & $29(2)$ & $14(1)$ \\
\hline \multicolumn{6}{|l|}{ Puesto } \\
\hline Auxiliar & 29 & : $48(14)$ & $28(8)$ & $14(4)$ & $10(3)$ \\
\hline General & 72 & $29(21)$ & $39(28)$ & $22(16)$ & $10(7)$ \\
\hline Especialista & 19 & $42(8)$ & $32(6)$ & $21(4)$ & $5(1)$ \\
\hline Jefe(a) de servicio & 16 & $19(3)$ & $43(7)$ & $19(3)$ & $19(3)$ \\
\hline \multicolumn{6}{|l|}{ Servicio de adscripción } \\
\hline Medicina interna ' & 43 & , $35(15)$ & $28(12)$ & : $21(9)$ & $16(7)$ \\
\hline Área crítica & 35 & ' 33 (12) & $29(10)$ & $29(10)$ & $9(3)$ \\
\hline Área quirúrgica & 24 & $38(9)$ & $46(11)$ & $8(2)$ & $8(2)$ \\
\hline Área pediátrica & 17 & $12(2)$ & $59(10)$ & $17(3)$ & $12(2)$ \\
\hline Consulta externa & 17 & $47(8)$ & $35(6)$ & $18(3)$ & $0(0)$ \\
\hline Total & 136 & $34(46)$ & $36(49)$ & $20(27)$ & $10(14)$ \\
\hline
\end{tabular}


Del análisis de algunos de los îtems de Percepción acerca de los cursos de educación continua, resalta la mirada que tiene el personal de enfermería respecto a: el 26\% (36) considera que los cursos nunca, casi nunca y, a veces, son útiles para desarrollar su actual trabajo; el 66\% (80) piensa que nunca, casi nunca o solo a veces, les permiten mejorar su ingreso económico $\left(r_{s}=0.250 p<0.003\right)$.

La antigüedad del personal de enfermería dentro del instituto se correlaciona, de manera negativa, con el número de cursos tomados $\left(r_{s}=-0.316, p<0.0001\right)$ esto es, a mayor antigüedad, menor número de cursos tomados; mientras que a mayor nivel académico se observa una correlacion positiva, ya que asisten a un mayor número de cursos $\left(r_{s}=0.265, p<0.002\right)$.
Esto es congruente cuando se exploran percepciones de la realidad que los encuestados viven en sus ambientes de trabajo y que tienen relación con condiciones administrativas. Así, cuando se les cuestiona cen el hospital de adscripción se elaboran programas de educación continua dirigidos a las necesidades que requiere?, se obtienen las siguientes percepciones: el 77\% (105) considera que nunca, casi nunca, o a veces; para el 66\% (90) ( $r_{s}=$ 0.469, p < 0.0001) la duración de los cursos no es el adecuado; el $61 \%(84)\left(r_{s}=0.554, p<0.0001\right)$ de los encuestados opina que los horarios no son afines, pues la mayoría se imparten en el turno matutino, cuadro ll.

Tabla 2. Perspectiva del personal de enfermería acerca de los cursos de educación continua. *

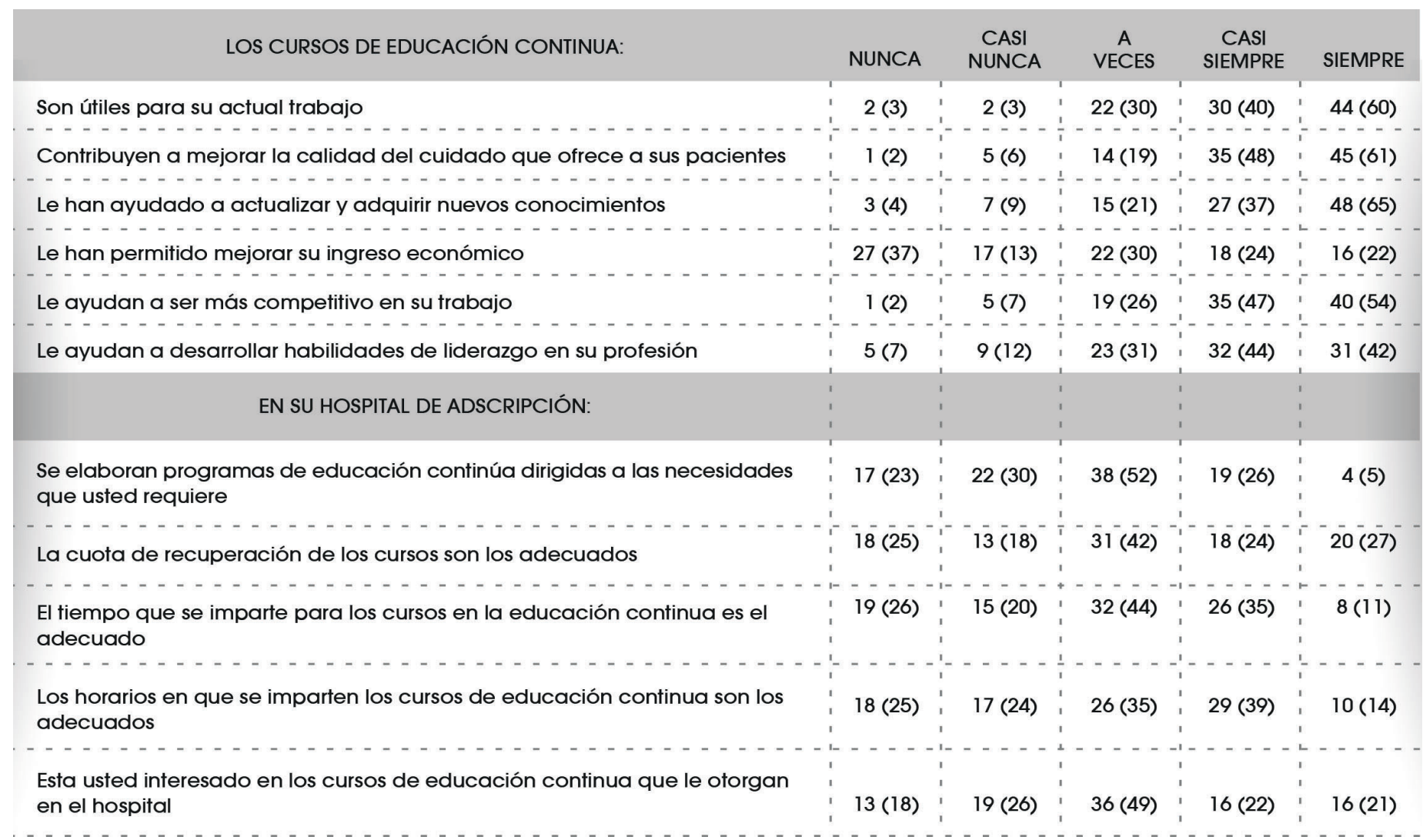

\section{* En porcentaje y frecuencia}




\section{DISCUSIÓN}

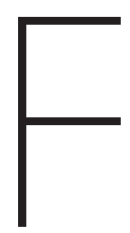

lores et al., en un estudio realizado en una institución pública, observaron que el tipo de personal de enfermería predominante era el de licenciatura, 60.9\%; por tipo de puesto, enfermería general 51.5\%, especialista 5\%; la no participación a cursos de educación continua fue del 1.5\%; la asistencia a cursos fue del 37\%. El $51 \%$ de este personal considera que los cursos son útiles para su trabajo actual. Al correlacionar, años de antigüedad con el número de cursos tomados, los autores no encontraron una diferencia estadísticamente significativa, esta información que difiere a la encontrada en nuestro estudio.

Se ha descrito que, entre los factores motivacionales del personal de enfermería que influyen en la asistencia a cursos de educación se encuentra: la falta de tiempo, la omisión de la difusión de los cursos de educación continua, más de una jornada laboral, desinterés personal por estudiar y la carencia de recursos económicos, ${ }^{8}$ factores con los que coincide este estudio.

Cadena et al., quienes realizaron un estudio para evaluar la capacitación del personal de enfermería en dos instituciones de salud, encontraron que el personal con más inasistencias fue el auxiliar, 30\%, mientras que el general, 18\% y las especialistas, 14\%. En este estudio los autores reportan que el número de cursos a los cuales asiste el personal de enfermería se ve relacionado con la edad, dato que concuerdan con el presente estudio; sin embargo, nuestro estudio difiriere respecto a los resultados de aceptación de los cursos de educación continua por parte del personal de enfermería, con el estudio de Cadena?

En un estudio realizado con enfermeras de Malaysia, se reporta como limitante para la asistencia a los cursos, la falta de oportunidades educativas por el alto costos y la falta de tiempo, ${ }^{4}$ limitantes que coinciden con nuestro estudio.

\section{CONCLUSIÓN}

I a literatura científica ha mostrado que tanto la educación continua, como la capacitación del personal de enfermería, repercute en un aumento en la productividad, en un número L menor de accidentes, mejores resultados en el paciente y en el trabajo y en un mejor clima organizacional, por lo que es importante la implementación de los cursos, y se tengan en cuenta las necesidades de aprendizaje del personal.

Los cursos de educación continua deben ser diseñados considerando las habilidades que se desea sean adquiridas por el personal de enfermería, que sean complementados con conocimientos técnicos de práctica laboral, con flexibilidad de horarios y tomados en cuenta para la obtención de estímulos económicos.

\section{1} MMN $\mathrm{WH}_{W}$ Enf Neurol Vol.16. No. 3 septiembre - diciembre 2017 


\section{REFERENCIAS BIBLIOGRÁFICAS}

1. Chiodelli SN, Do Prado ML. Educación continua en el trabajo: Nuevos desafíos para los profesionales de Enfermería. Invest. Educ Enferm. 2007; (25)1: 100-5.

2. Chong M, Sellick K, Francis K, Abdullah KL. What influences Malaysian nurses to participate in continuing professional education activities? Asian Nursing Research. 2017; 5 (1): 38-47. (Acceso 2016 noviembre 26). Disponible en: hittp://www.sciencedirect.com/science/article/pii/S1976131711600121

3. Meliem-Moctezuma J, Pérez-Castro V.J, Vázquez, Soło A.M. Identificación de la mala práctica de enfermería a partir de la queja médica. Revista CONAMED. 2013; 18 supl: S6-S16. (Acceso 2016 noviembre 05). Disponible en: $\quad$ http://www.dgdi-conamed.salud.gob.mx/ojs-conamed/index.php/ revconamed/article/view/130/154

4. Ross K, Barr J, Stevens J. Mandatory continuing professional development requirements: what does this mean for Australian nurses. BMC Nursing. (En línea) 2013; 12 (9): 7p. (Acceso 2016 noviembre 11); Disponible en: https:// bmcnurs.biomedcentral.com/articles/10.1786/1472-6955-12-9

5. Puntunet B.M, Domínguez B.A. La educación continua y la capacitación del personal de enfermería. Revista Mexicana de Enfermería Cardiológica. 200816 (3): 115-117. (Acceso 2016 octubre 29). Disponible en: http://www. medigraphic.com/pdfs/enfe/en-2008/en083g.pdf
6. Mendoza-Núñez V. Romo PR, Sánchez-Rodríquez M. Investigación. Introducción a la metodología. México: Universidad Nacional Autónoma de México; 2014

7. Flores PY, Alonso CM, Gómez MM. Factores que influyen en la participación del personal de enfermería en la educación continua (Tesis de Maestría).1999. (En línea). Universidad Autónoma de Nuevo León. (Acceso 2007 abril 13). Disponible en: http://eprints.uanl. $\mathrm{mx} / 6108 / 1 / 1080089074$. PDF

8. Hernández-Muñoz A, García-Aguiluz K, Ponce-Gómez G, Bernal-Becerril M, Rivas-Espinosa J. Factores motivacionales en el personal de enfermería que influyen en la asistencia a cursos de educación continua. Rev Enferm Inst Mex Seguro Soc. 2007; 15 (3): 125-128. (Acceso 2016 octubre 28). Disponible en: http://www.medigraphic.com/pdfs/enfermeriaimss/eim2007/eim073b.pdf

9. Cadena EJ, Olvera AS, Pérez LM, Balseiro AC, Matus MR. Evaluación de la capacitación de enfermería en dos institutos nacionales de salud Enfermería Universitaria ENEO-UNAM. 2012:19 (3):15-24. 